\title{
Nail Unit Blue Nevi: A 11-Case Series and Review of the Literature
}

\author{
Florence Dehavay $^{a}$ Sophie Goettmann ${ }^{b}$ Ines Zaraac Isabelle Moulonguet ${ }^{d}$ \\ Josette Andréa Marie Caucanas ${ }^{e}$ Robert Baran ${ }^{f}$ Bertrand Richert ${ }^{a}$ \\ for the Groupe Ongle de la Société française de Dermatologie
}

aSt Pierre, Brugmann and Children's University Hospitals, Université Libre de Bruxelles, Bruxelles, Belgium; ${ }^{b}$ Department of Dermatology, Hôpital Bichat - Claude Bernard, Paris, France; 'Dermatology Department, Groupe

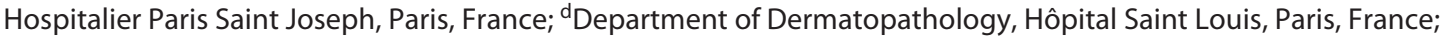

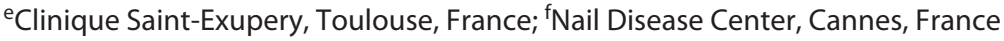

\section{Keywords}

Nevus · Blue nevus · Nails · Nail apparatus · Nail unit blue nevus · Subungual blue nevus · Nail melanoma

\begin{abstract}
Background: Blue nevus of the nail apparatus is a rare entity and only isolated cases are reported in the literature. Objective: The aim of this study was to better characterize blue nevus at the nail unit. Methods: Retrospective analysis of all nail unit blue nevi from the Nail Group of the French Society of Dermatology was compared to the literature. Results: Eleven cases were retrieved from 2002 to 2019 with an average age of 45 years. The majority were women (9/11) and acquired (10/11). Hands were more frequently involved $(9 / 11)$ with a predilection for the thumb and 2 cases were located on the hallux. Nail unit blue nevus mostly presented as a well-delimited blue spot of the lunula (9/11) and histologically was of the common type (10/11). There was no malignancy. Conclusion: Nail unit blue nevus is a rare asymptomatic benign entity, mostly acquired on the thumb or the hallux of women. The most frequent presentation is a painless blue spot on the lunula. Congenital blue nevi seem to only affect the paronychium. Main differential diagnosis is melanoma and histopathological examination is mandatory.
\end{abstract}

(c) 2020 S. Karger AG, Basel

\section{Introduction}

Blue nevi are a heterogeneous group of acquired or congenital benign dermal melanocyte proliferation $[1,2]$. The pathogenesis is not fully understood, but an embryological migration fault of the melanocytes from the neural crest to the epidermis is hypothesized $[2,3]$. The term "blue nevus" comes from the classic blue-gray hue related to the melanin location in the dermis and the reflection of short light wavelengths, in the blue spectrum, by melanin (Tyndall effect) $[1,2]$.

The lesions are solitary and more frequent in women [1]. There are many clinical and histological variants of blue nevi, but the major subtypes are the common and the cellular blue nevus $[1,2]$. Overlap between these subtypes is frequently reported with lesions sharing both histological features [1]. Although very rare, malignant transformation of a blue nevus or melanoma with histopathological features of blue nevus, the socalled "malignant blue nevus" have been described [4, 5]. Blue nevi occur mainly on the skin, but extracutaneous sites have been reported such as oral or genital mucosa, pulmonary or digestive tract, breast and prostate [1]. Another unusual location is the nail apparatus. The first case was described in 1984 by Soyer and Kerl [6]. 


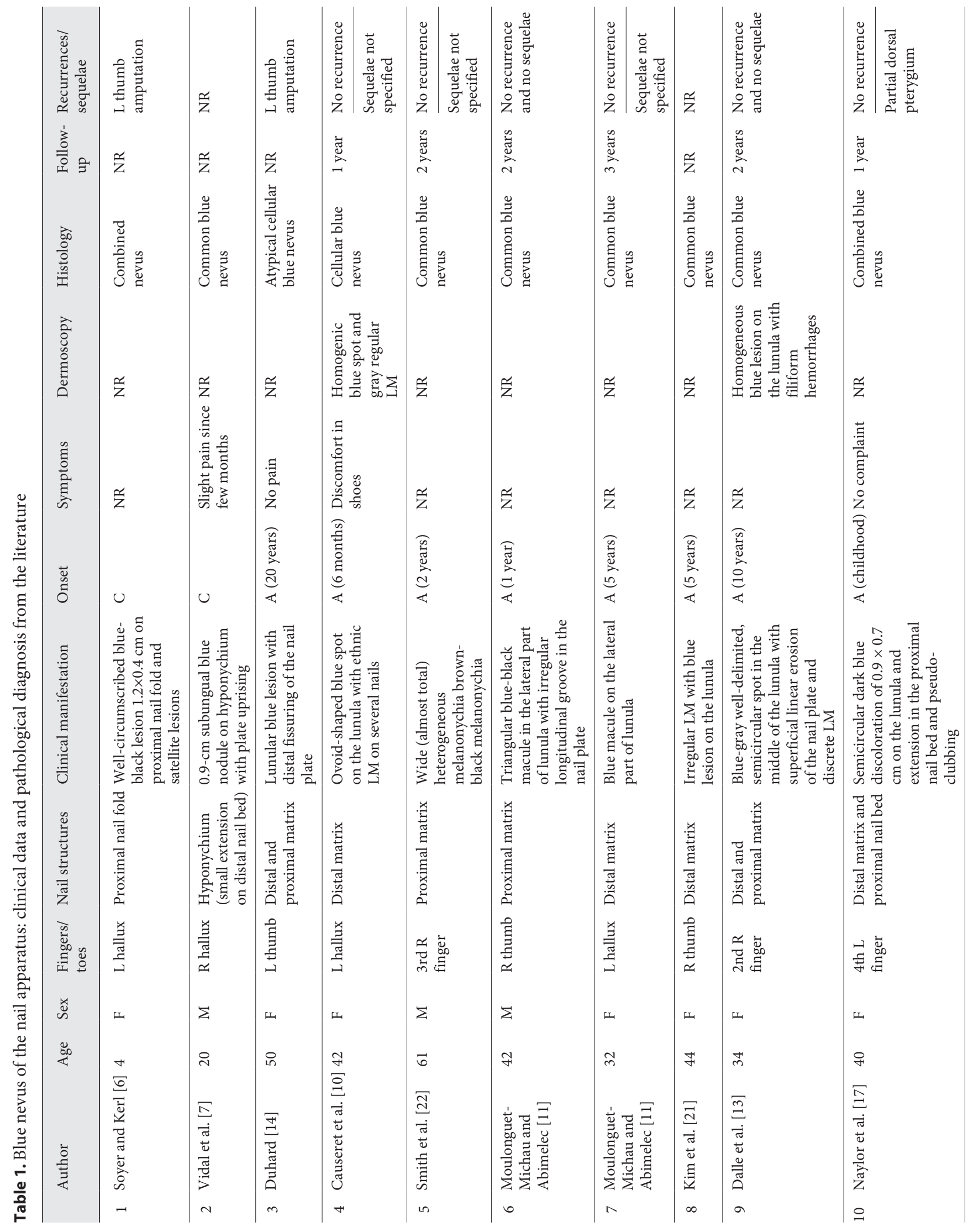




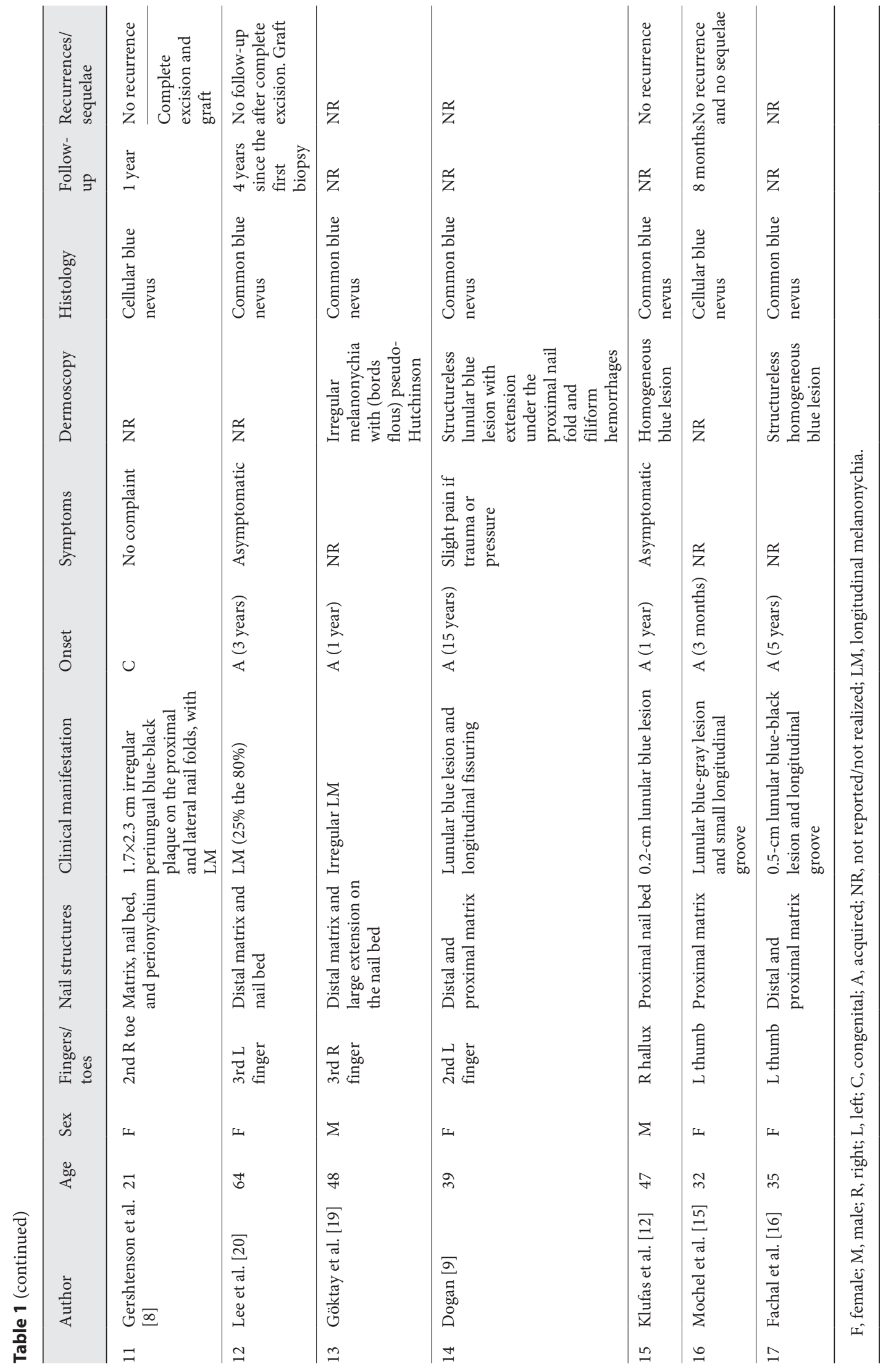




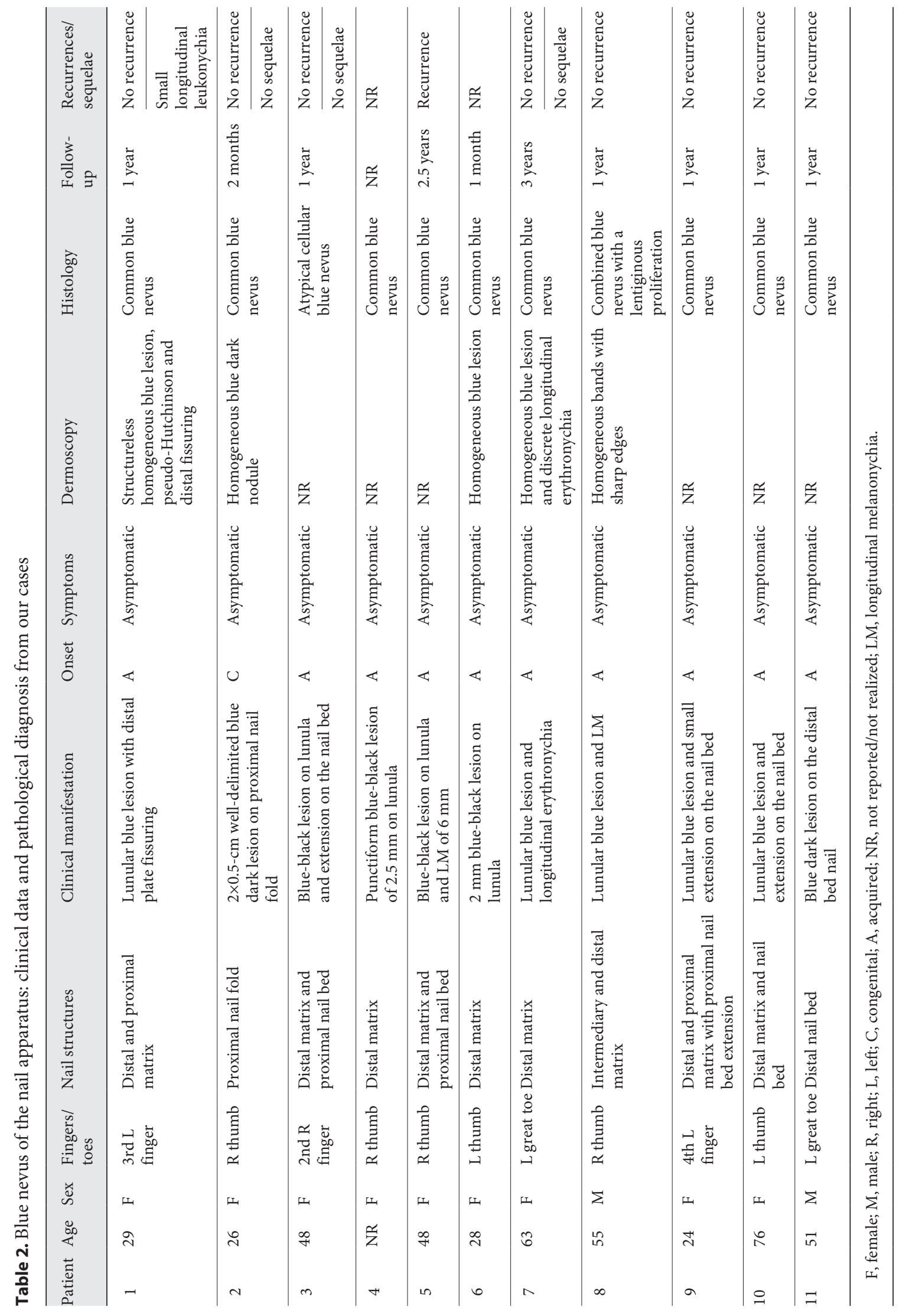



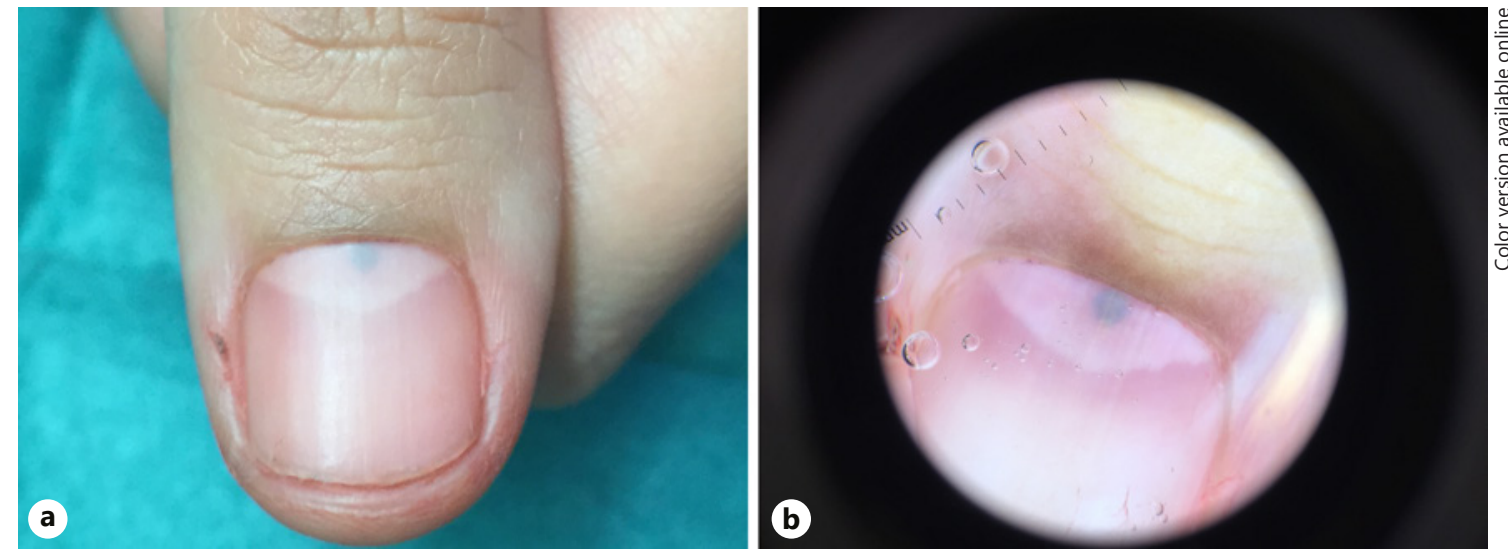

Fig. 1. Clinical (a) and dermoscopic (b) aspects of a typical nail unit blue nevus, presenting as a well-delimited blue spot on the lunula (patient 6).

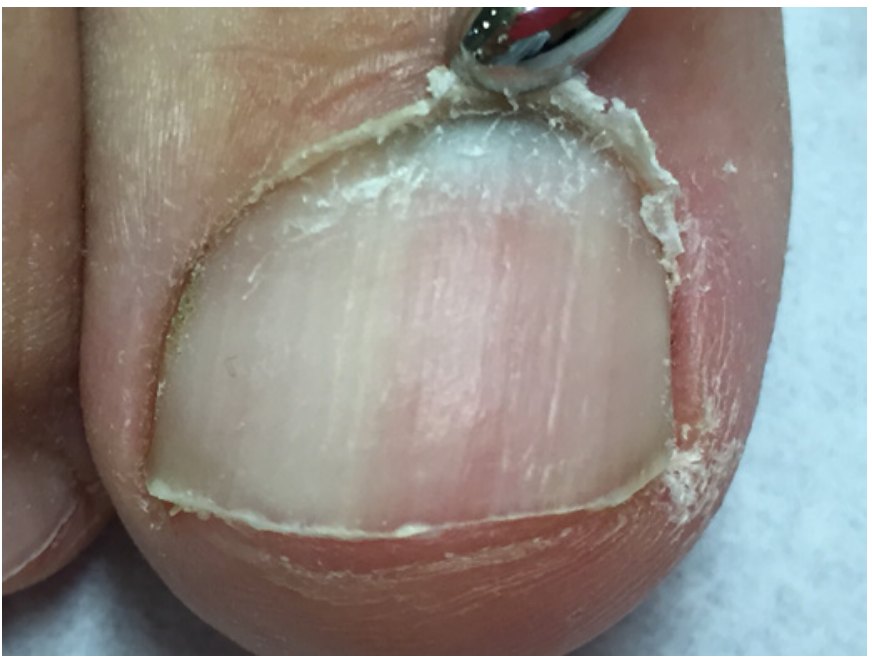

Fig. 2. Nail unit blue nevus with blue spot on the lunula associated with longitudinal erythronychia (patient 7).

Since then, a literature review mentions 16 other cases. Their clinical and histological characteristics are reported in Table 1.

\section{Objective}

The aim of the study was to collect all cases of nail unit blue nevi from the Nail Group of the French Society of Dermatology and to compare our data with those of the literature in order to better characterize this rare entity.

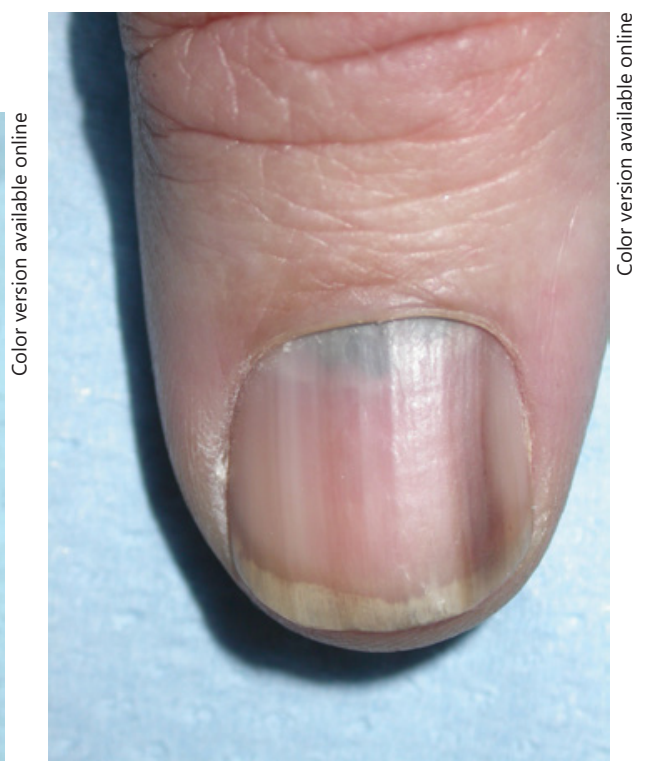

Fig. 3. Blue nevus presented as lunular blue lesion and LM (patient 8). LM, longitudinal melanonychia.

\section{Materials and Methods}

As a multicentric retrospective study, all cases of nail unit blue nevi were collected. Diagnosis was established by nail experts on the basis of clinical presentation and histological features. Surgical excision of the entire lesion was performed in all patients. All clinical information, clinical and histopathological pictures - and if possible, intra- and postoperative images - were reviewed. 


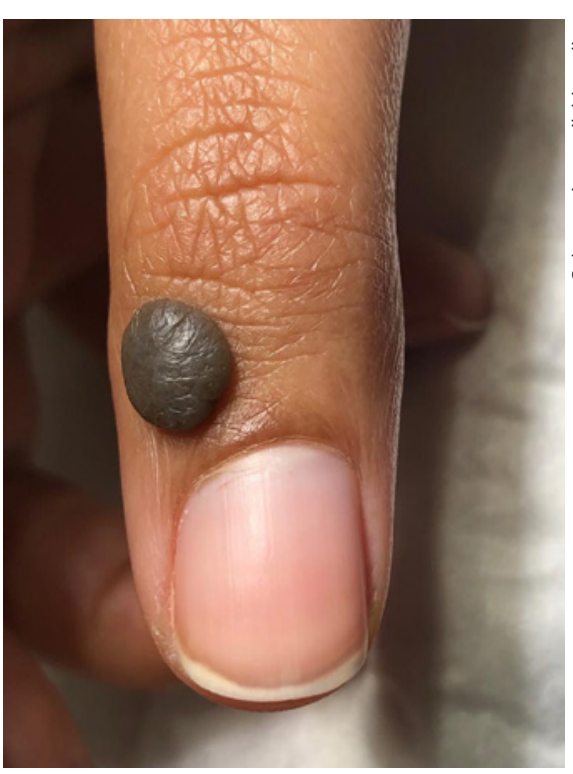

Fig. 4. Congenital blue nevus on the proximal nail fold of the right thumb (patient 2).
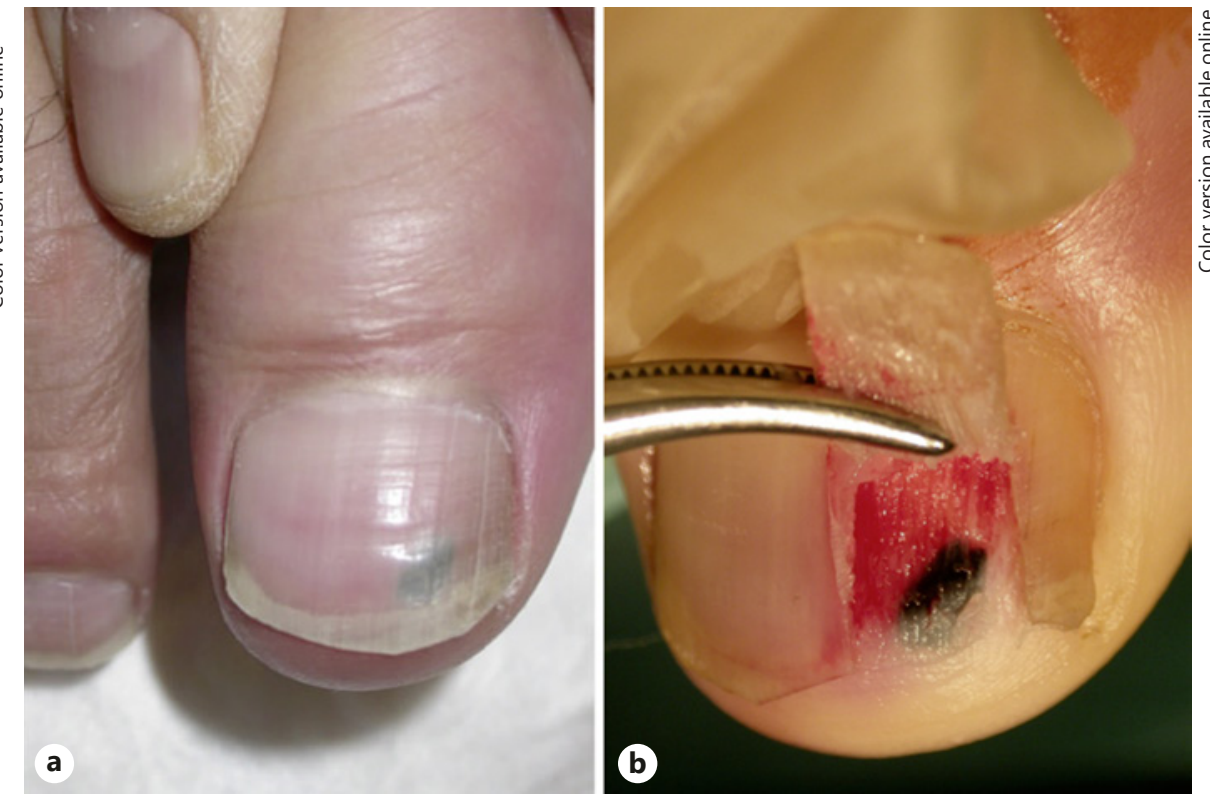

Fig. 5. Pre- (a) and intraoperative (b) pictures of nail unit blue nevus located on the distal nail bed (patient 11).
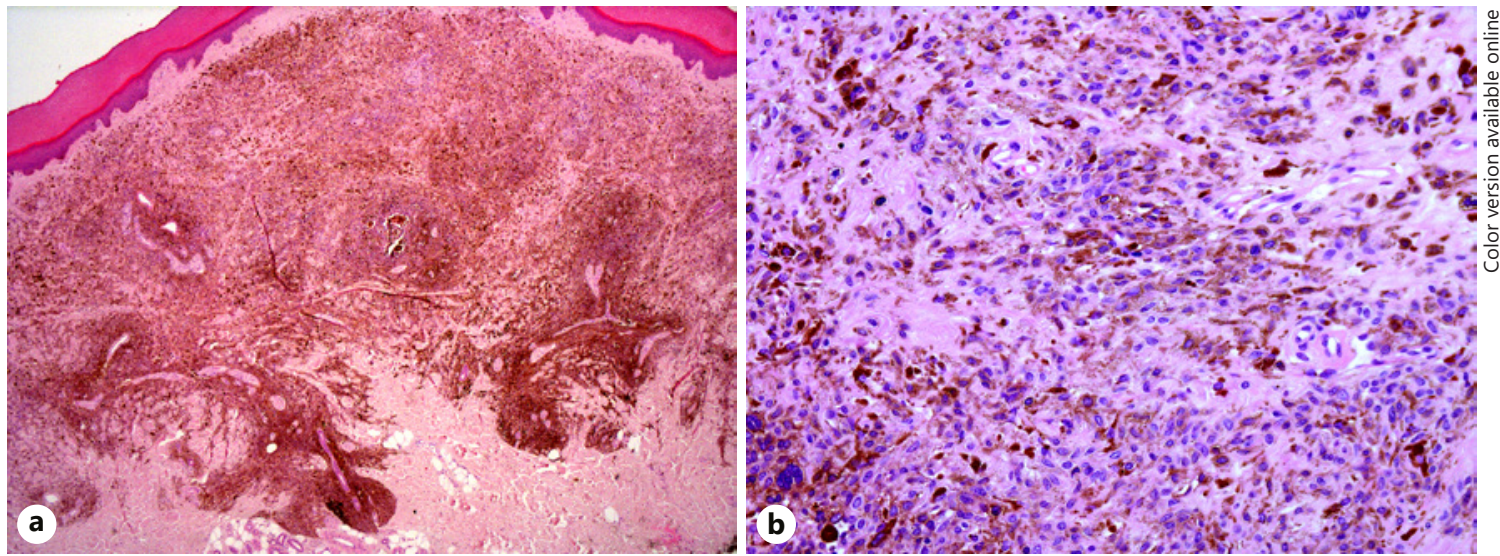

Fig. 6. a, b Histopathological features of common blue nevus. Dermal heavily pigmented proliferation of spindle melanocytes, clearly separated from the overlying epidermis. There is no melanocytic proliferation at the dermalepidermal junction, and no cellular pleomorphism, nor mitosis or tumoral necrosis in the dermis (patient 2).

\section{Results}

Eleven cases of nail unit blue nevi were retrieved from 2002 to 2019 with an average age of 45 years (24-76 years) (Table 2). The majority were women (9/11). Ten cases were acquired and one was congenital. Hands were more frequently involved (9/11) with a predilection for the thumb in 6 cases out of 9. Two nail unit blue nevi were located on the great toe. Clinically, blue nevi on the nail apparatus mostly presented as a well-delimited blue spot of the lunula (9/11) (Fig. 1). Two of them extended to the proximal matrix, and 1 case was associated with subsequent discrete distal fissuring of the plate. Another one was associated with longitudinal erythronychia (Fig. 2). Three extended to the proximal bed and 1 along the whole length of the nail bed. Two nail unit blue nevi were asso- 


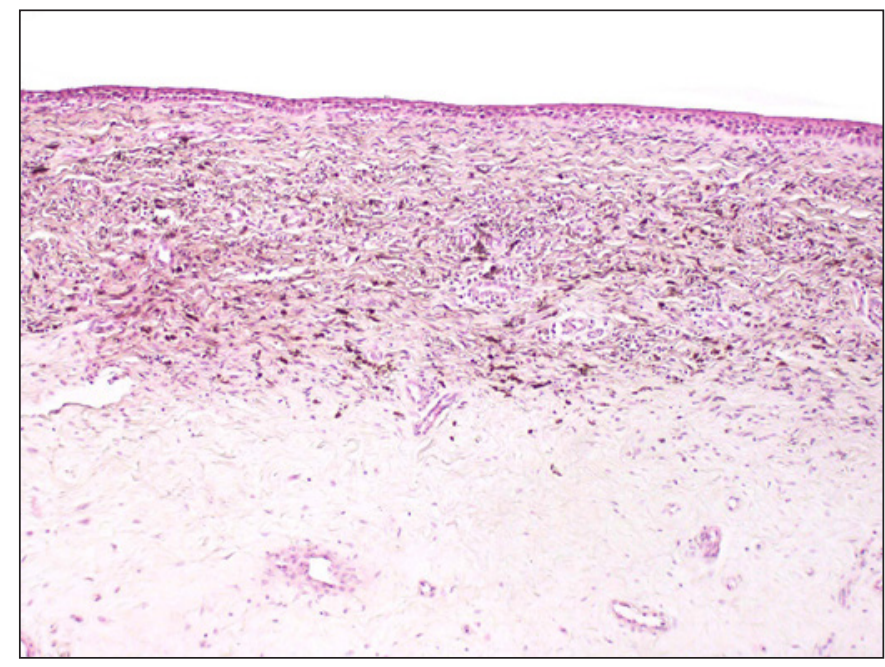

Fig. 7. Histopathological features of a combined blue nevus: discrete, noncontinuous lentiginous melanocyte proliferation above a dermal proliferation of pigmented, dendritic spindle melanocytes. This lesion was presenting clinically as lunular blue lesion and LM (patient 8). LM, longitudinal melanonychia.

ciated with longitudinal melanonychia (LM) (Fig. 3). One blue nevus was located on the proximal nail fold (Fig. 4) and 1 was isolated on the distal nail bed (Fig. 5).

Histologically, all blue nevi were of the common type (Fig. 6) except 1 cellular case showing atypical features without sign of malignancy. One of the common nail unit blue nevi associated with LM showed discrete, noncontinuous lentiginous proliferation of melanocytes in the basal cell layer of the matrix epithelium (Fig. 7).

\section{Discussion}

Blue nevi of the nail unit are rare with only 17 cases published up to now. All of them were isolated cases. With 11 cases, we herein report the largest series. Our results confirm the data from the literature (Table 3 ). The patients were mostly women which is consistent with the marked women's predominance found in the already published cases $(70.5 \%)$. There is no explanation for this female predilection. The majority of nail unit blue nevi is acquired, like on the skin [3]. Our series reported 1 congenital case, located on the proximal nail fold (Fig. 4). Soyer and Kerl [6] also reported 1 congenital case on the dorsal nail fold but with satellite lesions. The 2 others reported congenital lesions were on the hyponychium [7] and 1 spreading all around the nail unit [8]. There is no case of multiple nail blue nevi. None of our patients com-
Table 3. Comparison of literature cases to our case series

\begin{tabular}{|c|c|}
\hline Literature (17 cases) & Our series (11 cases) \\
\hline Female/male: 12:5 & Female/male: 9:2 \\
\hline 14 acquired/3 congenital & $\begin{array}{l}10 \text { acquired/1 } \\
\text { congenital }\end{array}$ \\
\hline 11 fingers: 5 thumb & 9 fingers: 6 thumb \\
\hline 6 toes: 5 great toe & 2 toenails: 2 great toe \\
\hline \multirow[t]{2}{*}{$5 \mathrm{LM}$} & $2 \mathrm{LM}$ \\
\hline & $\begin{array}{l}1 \text { longitudinal } \\
\text { erythronychia }\end{array}$ \\
\hline Matrix: 11 & Matrix: 5 \\
\hline Distal matrix: 5 & Distal matrix: 3 \\
\hline Proximal matrix: 3 & $\begin{array}{l}\text { Proximal and distal } \\
\text { matrix: } 1\end{array}$ \\
\hline Proximal and distal matrix: 3 & $\begin{array}{l}\text { Intermediary and } \\
\text { distal matrix: } 1\end{array}$ \\
\hline Matrix and nail bed: 2 & Matrix and nail bed: 4 \\
\hline Nail bed: 1 & Nail bed: 1 \\
\hline Proximal nail fold: 1 & Proximal nail fold: 1 \\
\hline
\end{tabular}

Hyponychium and nail bed: 1

Diffuse: 1

11 Common blue nevus

3 Cellular blue nevus

1 Atypical cellular blue nevus

2 Combined blue nevus

9 Common blue nevus 1 Atypical cellular blue nevus 1 combined blue nevus with a lentiginous proliferation

LM, longitudinal melanonychia.

plained of symptoms, but some authors reported discomfort, accentuated by pressure $[7,9,10]$.

The fingers are more often involved; $81.8 \%$ in our series and $64.7 \%$ in the other cases. Thumbs and halluces are the most common location. This could be explained by their larger matrix. In our series, the most frequent manifestation of nail unit blue nevi was a blue spot on the lunula (Fig. 1). This clinical presentation was also reported by Causeret et al. [10], Moulonguet-Michau and Abimelec [11], and Klufas et al. [12]. One of the cases with proximal matrix involvement was associated with distal plate fissuring. Several cases were also described with distal fissure and longitudinal groove $[9,11,13-16]$. Four patients had a blue nevus extending in the nail bed. Naylor et al. [17] 
Fig. 8. A tattooed distal nail bed (pencil lead) (a) and a painless glomus tumor (b) mimicking nail unit blue nevus.
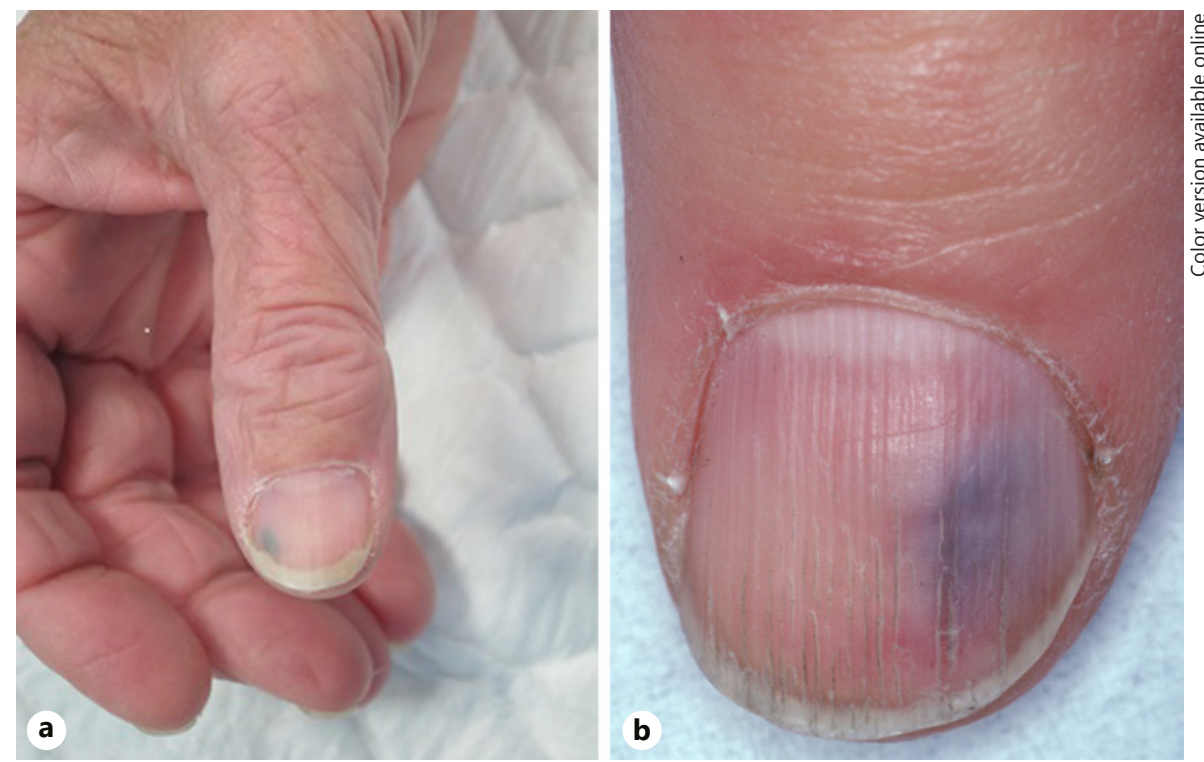

reported 1 case of blue nevus with a large extension on the nail bed associated with pseudo-clubbing.

Vidal et al. [7] reported a blue nevus on the hyponychium. We report the 2 nd case of nail unit blue nevus on the nail bed without matrix involvement [12]. The blue nevus predilection for the matrix, especially the distal one, could be explained by the density and activity of melanocytes in the different nail structures. Actually, in the matrix, melanocyte density is about $200 / \mathrm{mm}^{2}$, mainly dormant in the proximal matrix, dormant and activated in the distal matrix. In the nail bed, melanocytes are absent or only in very low density $\left(50 / \mathrm{mm}^{2}\right)$ and they are dormant [18]. Nevertheless, blue nevi are presumably linked to a failure of embryonic migration that could explain this unusual location. In the literature, 5 cases of nail unit blue nevi were reported with LM [10, 19-22]. Except for the case of Causeret et al. [10] who described typical multiple ethnic melanonychia striata, the other authors did not offer any explanation concerning these melanonychia. It is surprising to have melanonychia in blue nevi as this dermal lesion has no connection with the matrix epithelium. One may hypothesize that LM in the blue nevus results from melanocyte activation in the epithelium that lies above the dermal tumor. More rarely, it corresponds to a blue nevus combined with a lentigo as in 1 of our cases (Fig. 7). Unfortunately, the matrix epithelium of the 2 nd case with LM was damaged and could not be evaluated. We report the first case of nail unit blue nevus associated with erythronychia (Fig. 2). This might be explained by the mass effect of the tumor causing vascular congestion. The re- sidual postoperative erythronychia may be sequelae, as frequently observed after nail matrix surgery. Histologically, the vast majority of cases were common blue nevi (Fig. 6) with 1 combined blue nevus and 1 cellular case showing atypical features. There was no malignity. In the literature, common blue nevus was also the most frequent type but cellular, atypical cellular, and combined blue nevi were also described $[6,8,10,14,15,17]$. No case of "malignant blue nevi" at the nail apparatus has ever been reported. However, the most important differential diagnosis of blue nevus, as for all isolated pigmented lesion, is melanoma. Nodular melanoma of the nail bed should be ruled out when dealing with an isolated pigmented blotch of the nail apparatus as well as a melanoma originating in the matrix when associated with LM. We recommend to always perform a complete excision of the pigmented lesion to have an accurate histopathological diagnosis. Other clinical differential diagnoses are a pigmented foreign body and a painless glomus tumor (Fig. 8).

In conclusion, our series of 11 cases, the largest ever published, shows that nail unit blue nevus is predominantly acquired in women, on the thumb, or the hallux and is asymptomatic. The most frequent localization is the matrix with typically well-delimited blue spot on the lunula, associated or not with nail plate alteration. LM could be associated, mostly because of melanocyte activation. In the rare occurrence of a congenital nail unit blue nevi, it seems to only affect the paronychium. Even if no "malignant blue nevus" has been described, histopathological examination is mandatory to rule out melanoma. 


\section{Statement of Ethics}

The patients in this study gave written informed consent to publication of their case details.

\section{Conflict of Interest Statement}

The authors have no conflicts of interest to declare.

\section{Funding Sources}

No funding sources.

\section{Author Contributions}

All the authors have contributed to the conception of the work and the acquisition and analysis of the date. They have revised the content and approved the final version.

\section{References}

1 Murali R, McCarthy SW, Scolyer RA. Blue nevi and related lesions: a review highlighting atypical and newly described variants, distinguishing features and diagnostic pitfalls. Adv Anat Pathol. 2009;16(6):365-82.

2 Phadke PA, Zembowicz A. Blue nevi and related tumors. Surg Pathol Clin. 2009;2(3): 483-96.

3 Zembowicz A, Phadke PA. Blue nevi and variants: an update. Arch Pathol Lab Med. 2011; 135(3):327-36.

4 Mones JM, Ackerman AB. "Atypical" blue nevus, "malignant" blue nevus, and "metastasizing" blue nevus: a critique in historical perspective of three concepts flawed fatally. Am J Dermatopatho. 2004;26(5):407-30.

5 Sugianto JZ, Ralston JS, Metcalf JS, McFaddin CL, Smith MT. Blue nevus and "malignant blue nevus:" a concise review. Semin Diagn Pathol. 2016;33(4):219-24.

6 Soyer HP, Kerl H. European society of pediatric dermatology clinical case reports 1984. In: Baran R, Dawber RPR, Berker DAR, Haneke E, Tosti A, editors. Diseases of the nails and their management. 3rd ed. Oxford: Blackwell Science Ltd; 2001. p. 616-8.
7 Vidal S, Sanz A, Hernández B, Sánchez Yus E, Requena L, Baran R. Subungual blue naevus. Br J Dermatol. 1997;137(6):1023-5.

8 Gershtenson PC, Krunic A, Chen H, Konanahalli M, Worobec S. Subungual and periungual congenital blue naevus. Australas J Dermatol. 2009;50(2):144-7.

9 Dogan S. Subungual blue naevus presenting with elkonyxis. Hong Kong J Dermatol Venereol. 2016;24:87-91.

10 Causeret AS, Skowron F, Viallard AM, Balme B, Thomas L. Subungual blue nevus. J Am Acad Dermatol. 2003;49(2):310-2.

11 Moulonguet-Michau I, Abimelec P. [Nail unit blue melanocyte nevi: 2 case reports]. Ann Dermatol Venereol. 2004;131(11):984-6.

12 Klufas DM, Que SK, Berke A, Maryland BS. Acquired blue nevus of the nail bed. Dermatol Online J. 2017;23(2):13030.

13 Dalle S, Ronger-Savle S, Cicale L, Balme B, Thomas L. A blue-gray subungual discoloration. Arch Dermatol. 2007;143(7):937-42.

14 Duhard E. Naevus bleu cellulaire atypique. Nice: European Nail Society; 1998.

15 Mochel MC, Miller CJ, Rubin AI. Subungual pigmented tumor with onychodystrophy: challenge. Am J Dermatopathol. 2017;39(9): 707-8.
16 Fachal C, Pérez-Pérez LC, Allegue F, Calviño S. Subungual blue nevus. Actas Dermosifiliogr. 2018;109(1):88-90.

17 Naylor EM, Ruben BS, Robinson-Bostom L, Telang GH, Jellinek NJ. Subungual blue nevus with combined phenotypic features. J Am Acad Dermatol. 2008;58(6):1021-4.

18 Perrin C, Michiels JF, Pisani A, Ortonne JP. Anatomic distribution of melanocytes in normal nail unit: an immunohistochemical investigation. Am J Dermatopathol. 1997;19(5): 462-7.

19 Göktay F, Güneş P, Yaşar Ş, Güder H, Aytekin S. New observations of intraoperative dermoscopic features of the nail matrix and bed in longitudinal melanonychia. Int J Dermatol. 2015;54(10):1157-62.

20 Lee EJ, Shin MK, Lee MH. A subungual blue naevus showing expansile growth. Acta Derm Venereol. 2012;92(2):162-3.

21 Kim HS, Kim YJ, Kim JW, Yu DS, Yu DS. Subungal blue nevus. J Eur Acad Dermatol Venereol. 2007;21(2):271-2.

22 Smith DF, Morgan MB, Bettencourt MS. Longitudinal melanonychia. Arch Dermatol. 2003;139(9):1209-14. 